\title{
HOW RELIABLE IS AUTOMATIC OR MANUAL CALCULATION OF LUNG COMPLIANCE USING VENTILATOR READOUTS ? A MODEL STUDY
}

\author{
R. Hentschel ${ }^{1}$, N. Semar ${ }^{1}$, J. Guttmann ${ }^{2}$ \\ ${ }^{1}$ Neonatology/Intensive Care, Center for Pediatrics and Adolescent Medicine, University of Freiburg, \\ ${ }^{2}$ Division of Experimental Anaesthesiology, Department of Anaesthesiology, University of Freiburg, \\ Freiburg im Breisgau, Germany
}

Background and aims: Modern ventilators offer different tools for manual/automatic compliance (C) calculation, using measurement of pressure (PIP/PEEP) and tidal volume (Vt), but impact of varying ventilator settings and methodological limitations are not established.

To compare in a model study 4 different methods of $\mathrm{C}$ measurement: dynamic $\mathrm{C}$ (Cdyn), $\mathrm{C}$ calculated by the Mead-Whittenberger method (Cwitt), $\mathrm{C}$ calculated at quasistatic conditions (inflation hold (PIP) of 30 mbar(C-IH)), static C (Cstat) as gold standard.

Methods: Ventilation of 3 models of newborn lungs, representing different pressure/volume characteristics, with Dräger Babylog 8000plus. Study of varying combinations of ventilator settings, recording of: Cwitt as displayed by the ventilator; Cdyn calculated from Vt/(PIP-PEEP); C-IH calculated as Vt/30mbar, Cstat measured with Sensormedis2600. Statistics: Mean and standard deviation; Pearson linear regression; Kruskall-Wallis test.

Results: Unreliable results obtained with low ti and/or te. Analysis of pooled data: Cwitt higher, Cdyn lower than Cstat (both $\mathrm{p}<0.05$ ); at comparable settings (PIP 30mbar) tendency of C-IH to be lower and of Cwitt to be higher than Cstat; increasing deviation with increasing model lung $\mathrm{C}(\mathrm{C}-\mathrm{IH} / \mathrm{Cwitt}$ : lung $1+1.6 /-3.2 \%$; lung2 $+4.4 /-3.4 \%$; lung3 $+6.8 /-6.0 \%)$. Best correlation between C-IH and Cstat $\left(\mathrm{r}^{2}=0.998\right)$. Discrepancy results from underestimation of $\mathrm{Vt}(29.3 \%-43.2 \%$ for the 3 models $(\mathrm{p}<0.05))$.

Conclusions: $\mathrm{C}-\mathrm{IH}$ was superior to Cdyn and Cwitt and close to the standard Cstat. An important reason for deviation was underestimation of $\mathrm{Vt}$ by the Babylog. With short ti/te inaccuracy of pressure measurement may also be important. C-IH offers a good tool for bedside calculation of C. 